\title{
Average of Peak-to-Average Ratio (PAR) of IS95 and CDMA2000 Systems-Single Carrier
}

\author{
Vincent K. N. Lau, Member, IEEE
}

\begin{abstract}
Peak-to-average ratio (PAR) of a signal is an important parameter. It determines the input backoff factor of the amplifier to avoid clipping and spectral regrowth. In this letter, we analyze and compose the PAR of the downlink signal for IS95 and the CDMA2000 single-carrier systems. It is found that PAR of the transmitted signal depends on the walsh code assignment. Furthermore, we found that the PAR of CDMA2000 signal is always lower than the IS95 signal. Finally, PAR control by walsh code selection is proposed.
\end{abstract}

\section{INTRODUCTION}

$\mathbf{I}$ NDS-CDMA systems, the peak-to-average ratio(PAR) of the aggregate signal for multiple users is usually quite high (above $10 \mathrm{~dB}$ ). This puts a stringent requirement on the power amplifier and reduces the efficiency in the sense that a higher input backoff factor is needed before the peaks in the signal experience significance distortion due to power amplifier nonlinearity.

PAR analysis for OFDM has appeared in [1] and [2]. However, the PAR behavior of DS-CDMA is quite different from that of OFDM. In this letter, we try and analyze the PAR for the single-carrier CDMA systems. Both the IS95 (2G) and the CDMA2000 (3G) systems are compared. We found that the PAR of the forward link signals depend on the walsh code combinations. Furthermore, the PAR for CDMA2000 systems is always lower than that of IS95 systems. Finally, PAR control method by selective walsh code mapping is proposed and discussed.

This letter is organized as follows. In Section II, we define PAR. In Section III, we study the PAR dependency on walsh code selections for single-carrier IS95 and CDMA2000 systems. Finally, solution to reduce PAR for CDMA downlink signals is discussed in Section IV. We conclude with a brief summary of results in Section V.

\section{GENERAL PAR DEFINITION}

Intuitively, a peak of a signal $x(t)$ is given by the maximum of its envelope $|x(t)|$. However, for a continuous random process, $\max -x(t)$ - could reach infinity provided that the observation interval is long enough. Even in discrete random process where $\max |x(t)|$ is bounded, it may occur at very low probability which is not very useful in practice. Therefore, a more practical definition of peak is in probability terms given by the following.

Definition 1: A signal $x(t)$ is aid to have a peak at $x_{p}$ at cutoff probability $P_{c}$ if

$$
\operatorname{Pr}\left[|x(t)|<x_{p}\right]=P_{c}
$$

Manuscript received September 10, 1999. The associate editor coordinating the review of this letter and approving it for publication was Dr. A. Bahai.

The author is with the Department of Electrical and Electronic Engineering, University of Hong Kong, Pokfulam Road, Hong Kong (e-mail: knlau@eee.hku.hk).

Publisher Item Identifier S 1089-7798(01)03842-X

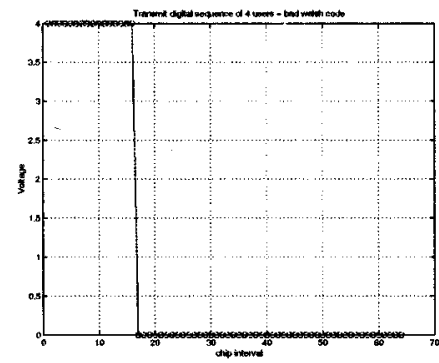

(a)

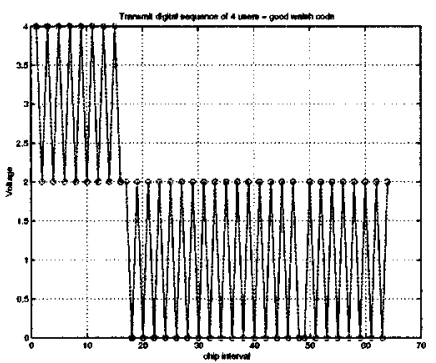

(b)
Fig. 1. An illustration of a good and a bad walsh code combination.

Therefore, the PAR of a random process $x(t)$ could be completely specified by its histogram.

\section{PAR FOR SINGLE CARRIER}

\section{A. Walsh Code Dependency}

Consider a coded bit duration $T_{b}$. The complex envelope of a CDMA downlink signal could be modeled by

$$
\tilde{s}(t)=\sum_{l=1}^{L}\left(\lambda_{l} P N_{I}(l)+j \lambda_{l} P N_{Q}(l)\right) p\left(t-l T_{c}\right)
$$

where $L$ is the number of chips per $T_{b}, \lambda_{l}$ is defined as the digital transmission sequence given by

$$
\lambda_{l}=\sum_{n=0}^{N_{u}-1} d_{n} w_{n}(l)
$$

where $N_{u}$ is the number of users; $d_{n}$ is the traffic data bit for user- $n ; w_{n}(l)$ is the $l$ th chip of walsh code assigned to user- $n$; $P N_{I}$ and $P N_{Q}$ are the pseudo-random short codes; and $p(t)$ is the transmission pulse shape. Index $l$ is used to indicate chip interval and index $n$ is used to indicate the $n$th user. $\lambda_{l}$ depends only on the choice of walsh codes and the applied data bits $\left\{d_{n}\right\}$. Due to the band-limited nature of the channel, the pulsewidth of $p(t)$ will generally span over several chip intervals, causing inter-chip interference at instants other than the ideal sampling times. Hence, a certain combination of walsh codes and data bits could result in a particular digital transmission sequence $\lambda_{l}$ that has a higher peak compared with the others. Let us illustrate the above idea with the following cases using the transmit pulse as specified in IS95.

Consider two different walsh code sets given by: 1) Walsh code set1: $=\left\{w_{0}, w_{32}, w_{16}, w_{48}\right\}, d_{n}(1)=\{1,1,1,1,1\}$. Walsh code set2: $=\left\{w_{0}, w_{1}, w_{33}, w_{49}\right\}, d_{n}(2)=$ $\{1,1,1,1,1\}$. The digital transmission sequences $\lambda_{l}(1)$ and $\lambda_{l}(2)$ are shown in Fig. 1(a) and (b), respectively. Because 


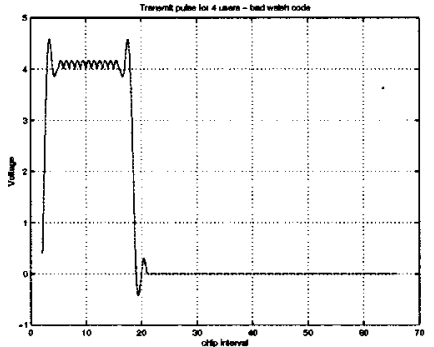

(a)

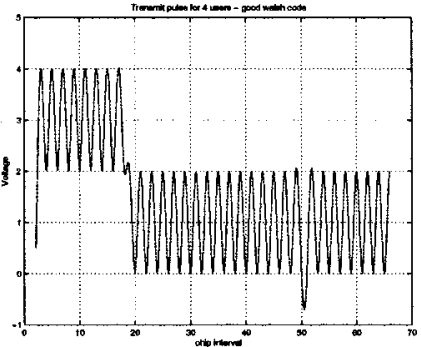

(b)
Fig. 2. Transmitted baseband signal of a good and a bad walsh code combination.

of the consecutive peaks at $l=14,15$, and 16 , the transmit pulse $p(t)$ would overlap and add constructively with each in Fig. 2(a). On the other hand, the bad combination of consecutive peaks are broken in the walsh set 2 and the resulting peak of the transmitted signal is smaller, as shown in Fig. 2(b). This explains the reason for the walsh code dependency of PAR.

\section{B. Comparison Between OS95 and CDMA2000 Systems}

It is shown by simulation that CDMA2000 system always has a lower PAR value than IS95 system (see Fig. 4). One difference between CDMA2000 and IS95 system employs QPSK modulation and complex spreading ${ }^{1}$ operation. We shall see that the PAR reduction of CDMA2000 is due to QPSK and complex spreading.

1) PAR Reduction Due to QPSK: The $I$ and $Q$ signals of IS95 downlink signal are given by

$$
\begin{gathered}
\tilde{s}_{I}(t)=\sum_{l} \lambda_{l} P N_{I}(l) p\left(t-l T_{c}\right) \\
\tilde{s}_{Q}(t)=\sum_{l} \lambda_{l} P N_{Q}(l) p\left(t-l T_{c}\right) .
\end{gathered}
$$

Peak position is totally determined by the digital transmission sequence. Hence, for a particular set of data set of data bits $\left\{d_{n}\right\}$ that causes $\lambda_{l}$ (and hence the $I$-signal) to peak at chip position $l$, the $Q$-signal, being affected by the same $\lambda_{l}$, is very likely to be large $I$ magnitude, as illustrated by the $I-Q$ plot of the IS95 CDMA complex envelope in Fig. 3(a).

On the other hand, if QPSK is used, $\lambda_{l}^{(I)}$ and $\lambda_{l}^{(Q)}$ are independent because they are driven by independent data bits $\left\{d_{N}^{(I)}\right\}$ and $\left\{d_{n}^{(Q)}\right\}$. Hence, $I$ and $Q$ signals become uncorrelated as illustrated by the spherical shape of the $I-Q$ plot in Fig. 3(b).

The overall peak of the complex envelope is given by $\sqrt{\left(\widetilde{s}_{I}^{2}(t)+\tilde{s}_{Q}^{2}(t)\right)}$. A large peak will occur only when both the I and Q signals are large in magnitude. For the IS95 system, $I$ and $Q$ signals are heavily correlated. For the CDMA2000 system, the $I$ and $Q$ systems are heavily uncorrelated. Hence, the probability of having a large overall peak for CDMA2000 system is smaller than the IS95 system, as illustrated in Fig. 3. Note that the $I-Q$ correlation of IS95 signal is due to the fact the different users use the same $P N_{I}$ and $P N_{Q}$ sequence. If there is only one single user, the $I-Q$ correlation will disappear.

${ }^{1}$ Real spreading refers to spreading the $I$-data signal with $P N_{I}$ and the $Q$-data signal with $P N_{Q}$. Complex spreading refers to spreading the complex data $(I+j Q)$ with a complex spreading sequence $\left(P N_{I}+j P N_{Q}\right)$.

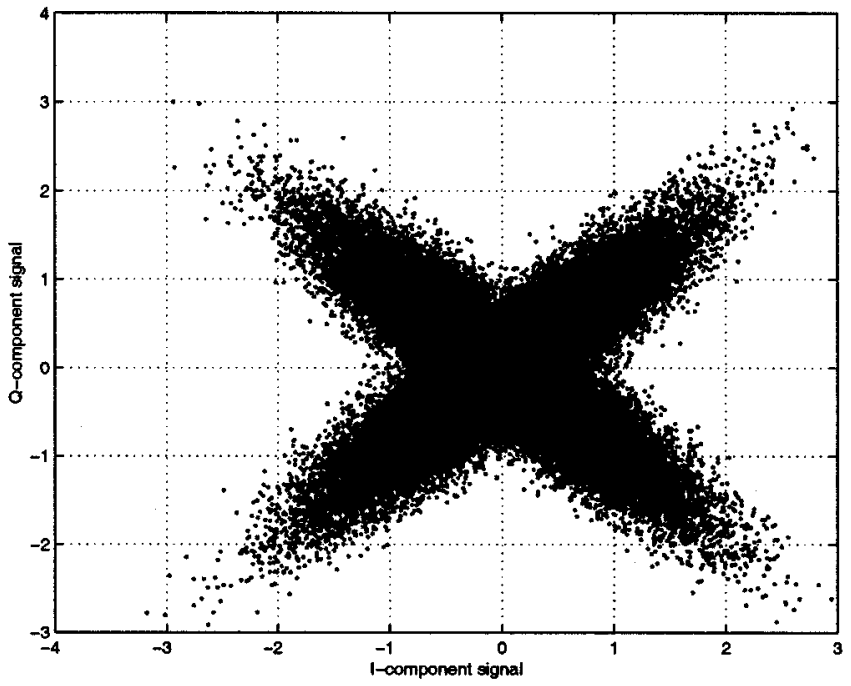

(a)

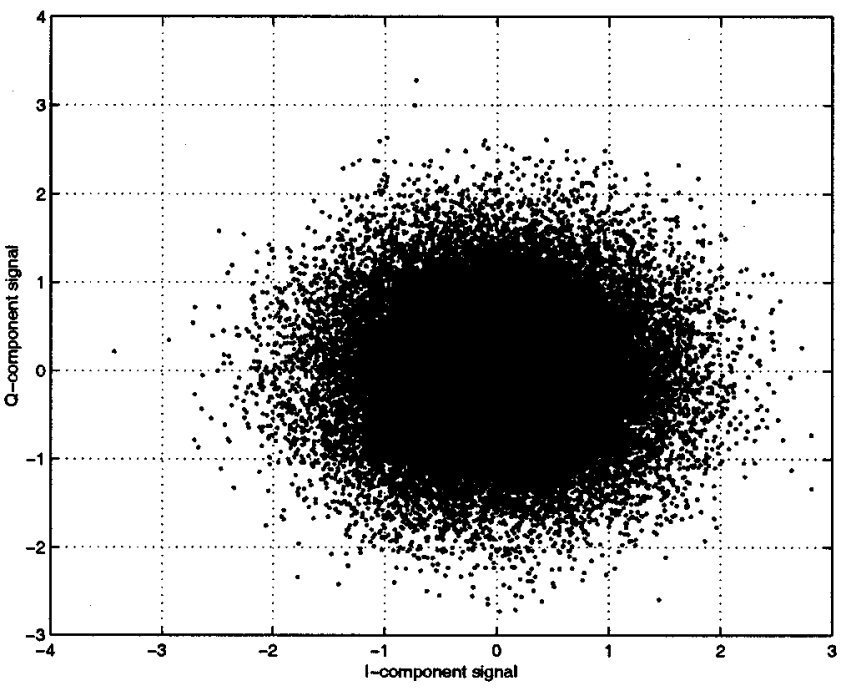

(b)

Fig. 3. Complex plot of $I-Q$ BPSK and QPSK signals for $N_{u}=10$. Signals are normalized to have the same rms power.

2) PAR Reduction Due to Complex Spreading: When QPSK with complex spreading is applied, the $I$ and $Q$ signals are given by

$$
\begin{gathered}
\tilde{s}_{I}(t)=\sum_{l}\left(\lambda_{l}^{(I)} P N_{I}(l)-\lambda_{l}^{(Q)} P N_{Q}(l)\right) p\left(t-l T_{c}\right) \\
\tilde{s}_{Q}(t)=\sum_{l}\left(\lambda_{l}^{(I)} P N_{Q}(l)+\lambda_{l}^{(Q)} P N_{I}(l)\right) p\left(t-l T_{c}\right) .
\end{gathered}
$$

In general, the gains applied to the $I$-channel data and the $Q$-channel data are different. The $I$-signal power and $Q$-signal power are given by

$$
\begin{aligned}
\sigma_{I}^{2}= & \lim _{T \rightarrow \infty} \frac{1}{T} \int_{-T / 2}^{T / 2} \tilde{s}_{I}^{2}(t) d t \\
= & \varepsilon\left[\left(\lambda_{l}^{(I)} P N_{I}(l)\right)^{2}\right]+\varepsilon\left[\left(\lambda_{l}^{(Q)} P N_{Q}(l)\right)^{2}\right] \\
& -\varepsilon\left[\left(\lambda_{l}^{(I)} \lambda_{l}^{(Q)} N_{I}(l) P N_{Q}(l)\right)\right]
\end{aligned}
$$


and

$$
\begin{aligned}
\sigma_{Q}^{2}= & \lim _{T \rightarrow \infty} \frac{1}{T} \int_{-T / 2}^{T / 2} \tilde{s}_{Q}^{2}(t) d t \\
= & \varepsilon\left[\left(\lambda_{l}^{(I)} P N_{I}(l)\right)^{2}\right]+\varepsilon\left[\left(\lambda_{l}^{(Q)} P N_{Q}(l)\right)^{2}\right] \\
& +\varepsilon\left[\left(\lambda_{l}^{(I)} \lambda_{l}^{(Q)} P N_{I}(l) P N_{Q}(l)\right)\right]
\end{aligned}
$$

where $\varepsilon(\cdot)$ is the expectation operator. Since $P N_{I}$ and $P N_{Q}$ are uncorrelated pseudo-random sequences, the third terms in $\sigma_{I}^{2}$ and $\sigma_{Q}^{2}$ are zero. Hence, $\sigma_{I}^{2}=\sigma_{Q}^{2}$ and potential $I-Q$ unbalance ${ }^{2}$ is removed.

\section{PAR CONTROL FOR Single CARRIER}

Several schemes have been proposed in [2] and [3] to deal with the PAR of OFDM signal by selective mapping or redundancy coding. For single-carrier DS-CDMA signal, the PAR is determined by the transmit digital sequence $\lambda_{I}^{2}$ and $\lambda_{Q}^{2}$ depend only on the walsh code. Hence, a simple way to control the PAR is to selectively assign walsh codes $\left\{w_{n}\right\}$ to each user so as to minimize the PAR. This is attractive because it does not require changes in the mobile station (MS) receiver. This is elaborated in the next subsection.

\section{A. Simulation Results}

The optimization problem is to select the best walsh codes for $N_{u}$ users that minimize the worst case PAR. Fig. 4 shows the difference in PAR (dB) of the IS95 and CDMA200 signals resulting from $\mathcal{W}_{\min }$ (the best walsh code selection) and $\mathcal{W}_{\max }$ (the worst walsh code selection), respectively. There is 1.5 and 2 $\mathrm{dB}$ PAR difference between the system using $\mathcal{W}_{\min }$ and $\mathcal{W}_{\max }$ walsh code for the IS95 and CDMA200 systems, Furthermore, there is a 1.5-2.5 dB reduction of PAR for CDMA2000 system. As the number of users per sector increases, the difference between the PAR's of the $\mathcal{W}_{\max }$ and $\mathcal{W}_{\min }$ decreased. ${ }^{3}$

The normal loading of current IS95 systems per sector is about 14 users. Therefore, the selective walsh code mapping method could contribute $1-2 \mathrm{~dB}$ reduction in PAR.

${ }^{2} I-Q$ balance is undesirable because it could raise PAR due to the loss of ergodicity.

${ }^{3}$ This is because the signal approaches a Gaussian distribution as $N_{u}$ increases.

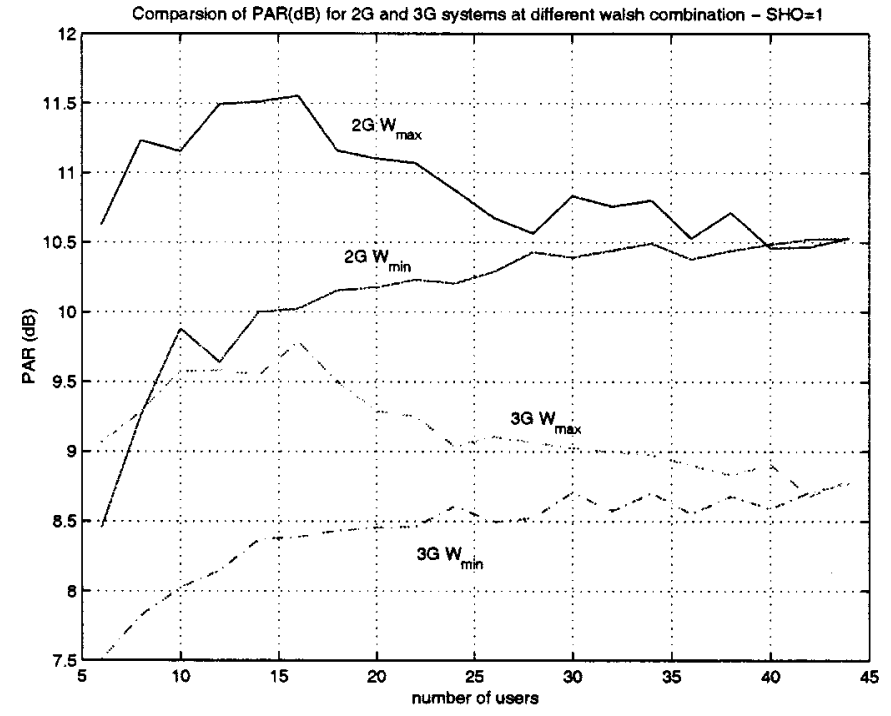

Fig. 4. Static comparison of PAR for IS95 and CDMA2000 systems with $\mathcal{W}_{\text {max }}, \mathcal{W}_{\text {min }}$ and $N_{u}$ as variables.

\section{CONCLUSION}

In this letter, we have discussed the PAR for the single carrier IS95 and CDMA2000 systems. We have found that the PAR depends on the walsh code combinations in the forward link signal. We also found that the PAR for CDMA2000 signals is always lower than of the IS95 signals. The reasons are due to QPSK modulation and complex spreading employed in CDMA2000. Finally, an algorithm to optimally select walsh codes (in PAR sense) have been proposed and its impact on PAR is discussed. In the static analysis, PAR gain of $1-2 \mathrm{~dB}$ is obtained.

\section{REFERENCES}

[1] R. O'Neill and L. B. Lopes, "Envelope variations and spectral splatter in clipped multicarrier signals," in Proc. 6th IEEE PIMRC'95, New York, June 1995

[2] B. M. Popovic, "Synthesis of power efficient multitone signals with flat amplitude spectrum," IEEE Trans. Commun., vol. 39, pp. 1031-1033, July 1991.

[3] S. Shepherd, J. Oriss, and S. Barton, "Simple coding scheme to reduce peak factor in QPSK multicarrier modulation," Electron. Lett., vol. 31, pp. 1131-1132, July 1995. 